Mots. Les langages du politique

$100 \mid 2012$

Chiffres et nombres dans l'argumentation politique

\title{
Portée et vertus des nombres dans les discours des Pères fondateurs de la République
}

Aude Dontenwille-Gerbaud

\section{(2) OpenEdition \\ Journals}

Édition électronique

URL : https://journals.openedition.org/mots/20980

DOI : $10.4000 /$ mots. 20980

ISSN : 1960-6001

Éditeur

ENS Éditions

\section{Édition imprimée}

Date de publication : 15 décembre 2012

Pagination : 43-56

ISBN : 978-2-84788-387-9

ISSN : 0243-6450

Référence électronique

Aude Dontenwille-Gerbaud, «Portée et vertus des nombres dans les discours des Pères fondateurs de la République », Mots. Les langages du politique [En ligne], 100 | 2012, mis en ligne le 15 décembre 2014, consulté le 23 avril 2022. URL : http://journals.openedition.org/mots/20980 ; DOI : https:// doi.org/10.4000/mots. 20980 


\section{Portée et vertus des nombres dans les discours des Pères fondateurs de la République}

De 1870 à 1882, la France vit toute une série d'événements : guerre francoprussienne, défaite de Sedan et proclamation de la République le 4 septembre 1870, siège de Paris, élections législatives de février 1871 et chambre non républicaine, Communes, Ordre moral, lois constitutionnelles de 1875 , République "opportuniste» à partir de 1879, naissance d'une opposition « socialiste» structurée. Les campagnes ne sont pas acquises à la République et il faut attendre 1879 (arrivée d'une majorité républicaine au Sénat et élection de Jules Grévy à la présidence) pour que la République ne soit plus menacée. Durant cette dizaine d'années, les Pères fondateurs de la Troisième République inaugurent une forme nouvelle d'intervention politique en se rendant régulièrement devant des publics populaires, tant à Paris qu'en province. On assiste à la naissance d'une figure historique, celle de l'orateur républicain, figure discursive de la République opportuniste. Quel que soit leur statut parisien, quelle que soit leur fonction d'élu ou encore leur importance au sein du mouvement républicain, les orateurs ne trouvent légitimité que dans le contact avec un public populaire. Tout se passe comme si le lieu de fondation de la République ne pouvait pas se suffire du théâtre parisien et des instances institutionnelles. Pour autant, ces orateurs ne se rendent jamais que devant des publics acquis à la République. Leur objectif n'est pas de convaincre des opposants, mais de former un public à devenir porte-parole, dans une France où la République fait encore peur. Les orateurs argumentent, s'adaptent à leurs publics dans une forme d'interaction largement retranscrite ensuite dans la presse. La parole des orateurs, les manifestations des publics interprétées par les sténographes, les comptes rendus de presse sont autant d'actes langagiers qui constituent l'événement discursif (Guilhaumou, 2006) fondateur de la Troisième République.

L'historien qui travaille en analyse du discours prend en compte un large corpus ouvert d'actes langagiers (retranscriptions des discours d'orateur, interprétation et retranscription des réactions des publics, comptes rendus de presse, correspondances, archives de police...). Plus précisément, il sera question ici 
de quelques extraits d'un «moment de corpus» de 164 discours $^{1}$ devant des publics populaires, prononcés entre 1871 et 1882 par Paul Bert, Louis Blanc, Jules Ferry, Charles Floquet, Léon Gambetta, Eugène Spuller. L'unité de genre permet les comparaisons malgré les thématiques et les longueurs diverses de ces discours. Les sténographes rendent compte des réactions des publics. Ce ne sont que des indices de la réception des discours. Nous n'aurons jamais accès à la certitude objective des paroles prononcées ni aux réactions effectives des publics. Toutefois, les sténographes qui accompagnent les hommes politiques et sont aussi, pour la plupart, sténographes officiels au Parlement, ont un niveau de formation très élevé et participent d'une déontologie professionnelle rigoureuse.

C'est justement sa technique (et la virtuosité ordinaire que son expérience exige) qui garantit la vérité d'un témoignage où le jugement est suspendu. Le sténographe semble ainsi doté de la capacité de transformer les faits en mots et ne s'autorise aucun mot sur ces faits ainsi traduits, ainsi transcrits. (Gardey, 2008, p. 46)

Ces manifestations, retranscrites avec «art» par les sténographes, sont à mettre en relation avec ce que donne à voir de son public l'orateur dans la matérialité de la langue. En prenant garde de ne pas essentialiser les réactions du public, il reste possible de rechercher trace dans les discours d'une expérience d'appartenance à un groupe, d'histoire partagée, de rituels d'appartenance caractéristiques des publics traditionnels (Dayan, 1992). Cette analyse s'inscrit dans une réflexion pragmatique sur les interactions où le destinataire n'est plus considéré comme passif (Kerbrat-Orecchioni, 2002, p. 173).

Au sein de la rhétorique argumentative républicaine de ce «moment de corpus », les nombres sont très présents. Certaines dates n'ont nul besoin d'être explicitées et fonctionnent comme topoï: le 2 décembre (chacun comprend la nuit sinistre du coup d’État de 1851), le 24 février (la révolution populaire par excellence de 1848), le 16 mai (la crise politique de 1877), pour n'en prendre que quelques-uns. Les nombres désignent parfois des acteurs majeurs comme les soldats de l'An II, le Manifeste des 60 (celui des candidatures ouvrières en 1860) ou encore les 363 (députés républicains opposés à Mac-Mahon). L'article VII représente aux yeux de tous la guerre contre les congrégations, identification majeure au combat pour la défense de la laïcité. Dénoncer les 45 centimes, c'est se battre contre le timbre imposé par l'Ordre moral qui tend à censurer la presse républicaine populaire. Les nombres, enfin, sont mobilisés comme effet rhétorique, entre rire et pathos.

Ces actes langagiers participent de l'expérience de fondation de la République, contexte qu'ils contribuent à modifier. Cette performativité du langage

1. Ces discours ne sont pas encore tous numérisés, ce qui interdit un traitement automatique d'ensemble. 
s'inscrit dans l'horizon d'attente (Koselleck, 1990) d'une République qu'un nombre qualifie, le Un. La notion méta-historique d'horizon d'attente permet de sortir d'une histoire linéaire pour prendre en compte l'analyse de l'emploi et de la signification des concepts politiques et sociaux. Ce Un constituant se réalise dans la rencontre même entre l'orateur et ses publics : se montrer homme régénéré donc libre, digne du suffrage universel, tout en héritant du bouillonnement des actes fondateurs de la Révolution. Les temps héroïques révolutionnaires sont clos, les Pères fondateurs de la Troisième République ne cessent de le répéter. Mais sans la «source authentifiante» (Maingueneau, Cossuta, 1995), il n'est point de fondation de la République.

Dans cet article, nous évoquerons tout d'abord les vertus légitimante et stigmatisante du nombre. Ce sont elles qui apparaissent d'emblée à la lecture de ces discours. Nous présenterons également un exemple de bataille de chiffres pour montrer comment le nombre devient argument d'autorité. Nous interrogerons ensuite la portée mobilisatrice des nombres utilisés dans les registres du rire et du pathos. Enfin, nous analyserons le fonctionnement discursif de l'Un et de l'indivisible : vertu unifiante fondatrice certes, mais portée par quels types d'arguments?

\section{Les vertus tour à tour légitimante ou stigmatisante du nombre}

Le nombre, dans les discours républicains, est d'abord utilisé comme topos, au sens de lieu commun, d'opinion partagée. Nous n'évoquons pas ici les topoï au sens rhétorique aristotélicien, logico-discursif (Amossy, 2000). Les nombres ne sont jamais des formes vides de raisonnement.

Ce n'est pas que la solution qui a prévalu doive nous causer une satisfaction sans mélange; elle ne répond que très imparfaitement, il faut bien le dire, à nos espérances, à nos aspirations et à ce besoin de sécurité que le Seize Mai avait rendu si impérieux (Marques d'adhésion). La France n'a-t-elle pas toujours à sa tête l'homme que de sinistres conseillers avaient poussé sur la pente qui mène à un Dixhuit Brumaire et à un Deux-Décembre? (Blanc, 1882, p. 281)

Ainsi s’exprime Louis Blanc dans une réunion électorale du 5 janvier 1878 à la veille des élections municipales². Dans ce discours (7 pages), le 16 mai

2. Depuis mars 1876, la Chambre des députés est composée d’une majorité de républicains. Le président de la République, Mac-Mahon, défend la droite conservatrice et cléricale. Le 16 mai 1877, il adresse une lettre au président du Conseil, Jules Simon. Il lui demande s’il a le sentiment d'avoir toujours «l'influence nécessaire pour faire prévaloir ses vues » sur la Chambre des députés. Les députés républicains considèrent cette lettre du 16 mai comme une atteinte au suffrage universel qu'ils représentent. Jules Simon démissionne. Mac-Mahon prononce la dissolution de la Chambre le 25 juin. 363 députés signent un manifeste d'union contre les menaces que fait peser le président de la République. La nouvelle Chambre élue en octobre, toujours majoritairement républicaine, refuse d'entrer en relation avec le nouveau cabinet ministériel. Le budget 
devient le syntagme nominal «le Seize Mai» qui ponctue régulièrement les phrases. Le 18 brumaire et le 2 décembre fonctionnent de la même manière, stigmatisant deux coups d'État, celui de Napoléon Bonaparte en 1799 (an VII) et celui du prince-président Napoléon III en 1851. Volonté du sténographe, de l'orateur, de l'éditeur, nous ne le saurons jamais, mais il est à noter que ces dates prennent ici la forme de mots, rédigés avec une majuscule, comme si le nombre constitué de chiffres s'effaçait dans l'argumentation. Peut-on analyser ces syntagmes comme des présupposés s'inscrivant dans une voix collective au sens de la polyphonie énonciative d'Oswald Ducrot (1984, p. 231-233)? En termes pragmatiques, nous pouvons évoquer un acte de langage formulé indirectement (Searle, 1982). Derrière l'acte de langage apparent de la question s'exprime un acte de requête : ne jamais renoncer à l'idéal républicain qui permettra d'en terminer avec le Second Empire. L'accumulation des dates substantivées renforce l'acte de requête. Louis Blanc pouvait attaquer successivement Napoléon ler, Napoléon III puis Mac-Mahon. L’argumentation reposait alors sur le risque que font courir à la démocratie des hommes ennemis de la cause républicaine. Évoquer des dates, ce n'est plus seulement juxtaposer des aventures individuelles, c'est proposer un récit historique, une chronologie de l'histoire de France qui s'inscrit dans le temps long, celui de la Révolution française et de son horizon d'attente. En termes argumentatifs, ces nombres substantivés remplissent donc une fonction cruciale, à partir d'une idée communément admise : malgré l'insécurité due aux ennemis de la République, la République dure et sait résister. De vertu stigmatisante, ces topoï deviennent en quelque sorte vertu légitimante, ce qui permet à Louis Blanc de conclure (quatre pages plus loin):

Au reste, quand on revoit le tableau des événements qui ont marqué les sept derniers mois de l'année 1877, il est une remarque qui vient naturellement à l'esprit. C'est celle-ci : de quelle vitalité ne faut-il pas que la République soit douée pour être sortie victorieuse des épreuves qu'elle vient de traverser? Ayons donc pleine confiance dans son avenir, mes chers concitoyens [...] sans nous dissimuler qu'elle a des ennemis implacables, et tout en nous gardant d'un excès d'optimisme qui risquerait d'endormir notre vigilance, tenons pour définitif son récent triomphe, et saluons-la immortelle (Triple salve d'applaudissements). (Blanc, 1882, p. 285)

Le nombre 7, ici simple marqueur de durée (sept mois), trouve sa force dans le récit historique du temps long, temps souligné par l'effet de pathos du début : «l'homme que de sinistres conseillers avaient poussé sur la pente de... », celle des 18 brumaire et 2 décembre toujours menaçante. L'ethos rhétorique de l'orateur se déploie dans cette mise en récit de l'histoire : Louis Blanc reste le républicain de 1848 qui garde confiance tout en restant vigilant.

Au sein de ce discours, le sténographe note une quinzaine de réactions du

n'étant pas voté, on craint, un temps, une déclaration d'état de siège pour lever l'impôt par décret. Les milieux d'affaire font pression et le centre-gauche revient au pouvoir. 
public qui ne semblent pas particulièrement liées à l'emploi d'une date substantivée. Les nombres, dans les discours de Louis Blanc, ne font que souligner des évidences communément partagées : les ennemis de la République abusent des coups de force et ne respectent pas la démocratie. La vertu stigmatisante sert la portée mobilisatrice.

Chez Victor Hugo, la construction rhétorique de soi donne au nombre une évidente vertu légitimante.

Vous êtes la vaillante jeunesse de l'humanité nouvelle. La vieillesse a le droit de saluer la jeunesse. Laissez-moi vous saluer. Laissez celui qui s'en va souhaiter la bienvenue à vous qui arrivez (Mouvement). Non, je ne me lasserai pas de vous rendre témoignage. J'ai été dix-neuf ans absent; j'ai passé ces dix-neuf années dans l'isolement de la mer, en contemplation devant les héroïques et sublimes spectacles de la nature, et, quand il m'a été donné enfin de revenir dans mon pays, quand je suis sorti de la tempête des flots pour rentrer dans la tempête des hommes, j'ai pu comparer à la grandeur de l'océan devant l'ouragan et le tonnerre la grandeur de Paris devant l'ennemi (Longs applaudissements). (Hugo, 1968, p. 361)

Ce discours de Victor Hugo est prononcé le 16 avril 1876 devant les délégués ouvriers français qui doivent se rendre à l'Exposition universelle de Philadelphie, le jour de Pâques, «communion populaire des nations »... Le nombre 19 n'est pas ici simple marqueur de durée. 1852-1870, ce sont les années de proscription et d'exil, les années du Second Empire. Le nombre 19 conforte la force illocutoire de l'ethos prédiscursif, celui du célèbre exilé de Jersey-Guernesey qui revient à Paris pour «faire halte entre l'exil et la tombe». Le marquage linguistique par le nombre agit sur le contexte qu'il contribue à modifier : l'interaction se veut passage de relais générationnel.

\section{Bataille de mots, bataille de chiffres. Le nombre comme argument d'autorité}

Lorsque Léon Gambetta s'exprime à Lille, le 15 août 1877, l'utilisation des nombres prend une large place :

Si le suffrage universel se bornait purement et simplement à décerner aux 363 le mandat qu'il leur avait confié il y a seize mois, sans aller au-delà, répétant simplement son verdict de 1876 , le résultat serait très considérable, très décisif, mais permettez-moi de dire qu'il ne serait pas suffisant.

Il ne serait pas suffisant à plusieurs points de vue : d'abord, parce qu'il ne constituerait pas une réponse et une protestation assez énergique contre l'acte du 16 mai ; ensuite, parce qu'il ne donnerait pas aux nouveaux élus une autorité complète pour en finir avec cette politique d'oscillations et de subterfuges (C'est cela! - Très bien! Très bien!-Applaudissements).

Aussi est-il permis de dire aujourd'hui, 15 août 1877, que c'est là une vérité que 
le suffrage universel a admirablement comprise : et les renseignements que nous possédons, qui sont absolument certains et circonstanciés sur les 533 circonscriptions du territoire de la République, nous permettent d'affirmer que le suffrage universel ne s'en tiendra pas à la réélection des 363, mais qu'il augmentera, dans une proportion considérable, le nombre des élus républicains du 20 février 1876 . J'ai parlé du chiffre de 400 , et on a cru que j'enflais mes espérances, que je grossissais mes prévisions. Non. Quand j'ai annoncé ce chiffre, je parlais avec la même conviction qu'au mois de février 1876 , lorsque, à Belleville, j'annonçais quel serait le résultat probable des élections qui devaient avoir lieu un mois après. Je disais alors que tout nous permettait de compter sur une majorité républicaine de 100 à 120 voix. On pensait que je grossissais le chiffre : la majorité a été de 200 voix (Salves d'applaudissements. - Bravos répétés). (Gambetta, 1881, VII, p. 222-223)

Sur les 263 mots prononcés ici par Léon Gambetta, nous pouvons compter 15 nombres, majoritairement rédigés sous forme chiffrée.

Nous retrouvons les topoï des 363 et du 16 mai pour renforcer l'argumentation. Le consensus est facile à dégager puisque tout le public républicain ne peut que réprouver le 16 mai et soutenir les 363 députés hostiles à Mac-Mahon.

Des durées et des dates précises s'inscrivent dans le récit de l'histoire : 1876, 1877, 15 août 1877, 2 o février 1876, avec cette fois un net effet d'insistance par l'utilisation des syntagmes : «il y a 16 mois », « aujourd'hui, 15 août 1877 », «au mois de février 1876 ». L'argumentation sur la prédiction réussie est, quant à elle, soulignée par les temporalités : «je disais alors... ", « on pensait que... », «la majorité a été... ». L'utilisation politique de nombres marquant des quantités s'inscrit dans le cadre d'une véritable bataille sur les «chiffres»:100, 200 voix ou encore 533 circonscriptions pour justifier les nouvelles prédictions («j'ai parlé du chiffre de $400 »$ ).

Le suffrage universel est l'acteur du récit historique souligné par les dates. Le «nous», celui du parti républicain, est l'auteur de son interprétation et trouve sa légitimité dans sa connaissance des 533 circonscriptions : la précision du nombre fortifie l'argumentation dont l'objectif est de rendre crédible ce que pense le «je », cette fois Gambetta lui-même et ses prédictions. La légitimation de cette prédiction passe également par la stigmatisation de l'adversaire, ce «on » qui finalement ne connaît pas suffisamment le terrain et, en quelque sorte, ne sait pas compter. D’après le sténographe présent, cette argumentation où l'utilisation des nombres est importante suscite globalement des applaudissements nourris. Mais le sténographe ne note pas de réactions particulières à l'évocation de ces nombres.

\section{Du rire au pathos : portée mobilisatrice du nombre?}

L'art oratoire des leaders républicains recherche également le rire, et le nombre se veut alors ironie et dérision. Entre autres exemples, l'hilarité des publics 
est notée par le sténographe lorsque Charles Floquet évoque les ouvriers de la $11^{\mathrm{e}}$ heure (les républicains un peu neufs) dont la vertu clivante permet $a$ contrario de susciter l'adhésion unificatrice de son public. L'orateur emploie cette expression le 29 août 1880 à Rouen au banquet des Chambres syndicales, le 12 septembre 1880 au Havre, là encore au banquet des Chambres syndicales ouvrières, et le 24 octobre 1880 à Valence devant 500 personnes réunies par voie de presse. Les réactions notées semblent identiques partout, et ce quel que soit le sténographe présent. L'interaction entre Charles Floquet et ses publics prend parfois l'allure de la routine, ficelle du métier d'orateur, qui marche au demeurant comme ainsi à Rouen, le 29 août 1880 :

Quant à vous, mes chers concitoyens, quand vous comparez la situation dans laquelle se trouvait la République il y a un an et la situation où elle se trouve aujourd'hui, non, non, vous n'êtes pas découragés. Vous sentez bien que le progrès se fait; vous n'êtes pas fatigués, quoique vous ne soyez pas des ouvriers nouveaux dans la République, quoique vous ne soyez pas des ouvriers de la $11^{\mathrm{e}}$ heure, quoique vous soyez même, je le crois, et, en cela je vous approuve, des ouvriers qui demandez qu'on supprime la $11^{\mathrm{e}}$ heure... de travail (Rire général et adhésion unanime). Vous êtes les ouvriers de la première heure, et j'en vois un, en face de moi, qui a été, il y a longtemps déjà, un ouvrier de la première heure... (Vive approbation et cris répétés de : Vive le doyen!) Vous n'êtes pas fatigués de vos longues luttes pour la République, mais, ayant travaillé pendant les dix heures précédentes, vous vous croyez, avec raison, assez forts pour vous passer du concours onéreux des ouvriers de la onzième heure (Rires et applaudissements). (Floquet, I, p. 398)

Jean Maillet le souligne (2011, p.101), l'expression (déjà présente dans l'Évangile de Matthieu) prend une tournure particulièrement ironique dans les mondes politique et social. Très employée, elle garantit donc un certain succès oratoire. D'après les retranscriptions, le public s'en amuse à chaque fois. En est-il de même du nombre utilisé comme pathos?

Et savez-vous à quel chiffre s'élevèrent, quand la fumée du combat était à peine dissipée, les dénonciations dictées par les haines personnelles et par la vengeance? Il y en eut 399823 (Sensation profonde). Vous devinez ce que devait contenir de mensonges ce chiffre assassin. Qu'on s'étonne, après cela, si des erreurs sanglantes furent commises, si pour ne citer qu'un exemple, trois malheureux furent assassi... fusillés,

Une voix. - Dites le mot.

Eh bien, oui, assassinés (Applaudissements), sous le nom de Jules Vallès, et si sur 38000 personnes arrêtées, il y eut, suivant le rapport du général Appert, 20000 ordonnances de non-lieu. Un fait qui ne saurait être trop médité montre où peuvent conduire les procès qui donnent les vainqueurs pour juges aux vaincus. (Blanc, 1882, p. 378)

Il va de soi que les nombres ne sont pas ici simple marque quantitative. Dans ce discours à visée persuasive prononcé à Perpignan le 13 octobre 1879 
(Louis Blanc revendique l'amnistie pleine et entière des communards), les nombres fonctionnent comme éléments émotionnels, intimement liés à ce que Patrick Charaudeau (2000, p.131) analyse comme "savoir polarisé autour de valeurs socialement constituées». Les blessures de la Commune ne sont pas refermées. Les deux nombres, " 399823 dénonciations» et « 20000 ordonnances de non-lieu », stigmatisent certes les tribunaux militaires, mais plus encore les haines et vengeances personnelles. L'exemple porté par ces deux nombres, permet à l'orateur d'utiliser le pathos pour s'inscrire dans l'horizon d'attente des valeurs républicaines. En tant que républicain, il sait ne pas être suivi par Léon Gambetta, Jules Ferry, Paul Bert qui n'acceptent qu'une amnistie partielle (la Commune fait encore peur dans les campagnes). Mais dans le même temps, socialistes et communards commencent à construire l'histoire de la Commune. Or, Louis Blanc ne l'a pas approuvée en son temps et s'est même publiquement désolidarisé de ses égarements dans une lettre adressée au Figaro en juin 1871 (Aprile, 2005). Les nouveaux récits socialistes ne cessent de rappeler «la lâcheté de Louis Blanc». Dans ce discours, la portée unificatrice des nombres au sein de l'argumentation se manifeste par la «sensation profonde » et les « applaudissements » du public notés par le sténographe.

Quelle que soit la réécriture a posteriori de cette forme d'interaction, c'est l'union du peuple républicain qui est en jeu, projet fondateur d'une République qu'un nombre mythique incarne : l'Un.

\section{Vertu unifiante fondatrice : quels types d'arguments?}

Pour les Pères fondateurs de la République, il ne peut exister « une question sociale ", précisément parce que la République ne peut être qu'Une et indivisible (Nicolet, 1982, p. 251). La bataille pour le Un est primordiale.

Léon Gambetta lance sa célèbre «phrase » au Havre, le 18 avril 1872, au cours d'un discours relativement long :

Tenons-nous en garde contre les utopies de ceux qui, dupes de leur imagination, ou attardés dans leur ignorance, croient à une panacée, à une formule qu'il s'agit de trouver pour faire le bonheur du monde. Croyez qu'il n'y a pas de remède social, parce qu'il n'y a pas une question sociale. Il y a une série de problèmes à résoudre, de difficultés à vaincre, variant avec les lieux, les climats, les habitudes, l'état sanitaire, problèmes économiques qui changent dans l'intérieur d'un même pays; eh bien! Ces problèmes doivent être résolus un à un et non par une formule unique. (Gambetta, 1881, II, p. 263)

L'attaque est claire. Le public du Havre est composé de 600 convives, parmi lesquels des conseillers généraux et municipaux, des représentants de la presse républicaine. Le public ne semble pas réagir. Le sténographe ne note aucune réaction durant ce passage niant l'existence d'« une question sociale». 
Il est toujours difficile d'interpréter les silences des publics. S'agit-il de réprobation, d'étonnement, d'inquiétude?

Cette phrase suscite de nombreux mécontentements dans la presse, parmi lesquels, celui de Louis Blanc, dans le Rappel du 25 avril. La République française répondra au Rappel. L'idée de Léon Gambetta, lancée lors du discours du Havre, devient instantanément objet de débat. Peut-on, dès lors, évoquer une petite phrase? Caroline Ollivier-Yaniv et Alice Krieg-Planque (2011) ont montré comment cet objet petite phrase s'inscrit dans des dimensions énonciative, médiatique, politique et communicationnelle. Nous avons là une forme brève d'énoncé détachable coproduit par les médias et les politiques (Krieg-Planque, 2011, p. 29). Dans ces années 1870-1880, la presse écrite s'articule déjà avec l'oralité des discours, non pas en simple écho, mais dans une dynamique qu'il convient de problématiser comme l'entrée dans l'ère moderne des médias, au sens où peuvent l'analyser Gilles Feyel (2007) ou encore Dominique Kalifa (2001).

Léon Gambetta laisse le temps s'écouler. Il faut attendre cinq mois, le 30 septembre 1872 à Bonneville, pourvoir réapparaître le thème. Entre-temps, Gambetta a prononcé huit autres discours sans mentionner cette question sensible. Il commence son discours en évoquant « toute l'effusion de son âme» et relance l'idée des questions sociales au pluriel.

\footnotetext{
Car, de quelque côté qu'on prenne la question, qu'il s'agisse des questions politiques ou des questions sociales, de quelque côté qu'on tourne et retourne le problème, de quelque sens qu'on l'envisage, on aboutit constamment à cette solution qu'il n'y a rien à tenter, rien à essayer, rien à espérer, entendez-le bien, tant qu'on n'aura pas constitué pour tous, avec le concours de l'État, des départements et des communes, la dotation de l'instruction générale de notre pays... (Gambetta, 1881, III, p. 143)
}

La petite phrase a disparu. «Il n'y a pas une question sociale» ne peut plus se dire. Léon Gambetta profite du combat pour l'instruction gratuite, laïque et obligatoire, unanimement partagé par l'ensemble des républicains, pour rappeler au détour d'une phrase qu'il y a des questions sociales. Le biais d'autres questions sensibles sert alors de tremplin.

Dans la disparition du «un » se joue l'ensemble du discours républicain opportuniste à l'égard du socialisme. À terme, pour Léon Gambetta et ses partisans, la République devra reposer sur deux grandes forces politiques : une force conservatrice et une force de progrès. Dans cette logique, le socialisme ne peut plus faire sens. II n'est autre que la version sociale de la République ellemême. La disparition du terme même de socialisme dans le champ discursif de la République triomphante ne fait que dire la tension paradoxale entre l'Un et le multiple. L'évitement du terme, corrélatif à l'annonce de la fin des temps héroïques et de l'achèvement de la Révolution française, revient à en revendiquer l'héritage, tant spontané et populaire (les temps héroïques) qu'intellectuel (la science politique). En ce sens, cet acte de langage ne peut s'analyser 
sans référence à l'histoire langagière du concept socialisme (Branca-Rosoff, Guilhaumou, 2003).

Pierre Rosanvallon (1998, p. 31) analyse cette tension politique comme celle d'un décalage entre le «peuple-principe» qui universalise l'entité nationale et la vérité du lien social. Dans l'interaction, cependant, entre l'orateur républicain et son public populaire, peut-on analyser discursivement cette tension? Léon Gambetta s'exprime par exemple à Lyon, le 28 février 1876, devant un large public populaire, Lyon devenue capitale rouge depuis 1871 , siège de nombreuses émeutes et grèves menées par des ouvriers de métiers, peuple d'avant-garde, libre penseur, difficile à contenir, et avec lequel la République naissante ne doit pas tergiverser (Lequin, 1977; Perrot, 1984).

[...] que signifie la majorité élue le 20 février? Elle signifie que la plus grande partie de la nation s'est prononcée pour la défense, pour l'affermissement et le développement de la Constitution républicaine, c'est-à-dire pour la stabilité, pour l'ordre légal et la liberté progressive. Or, ce qu'il faut regretter, ce n'est pas le chiffre élevé de la majorité, c'est qu'il n'y ait pas l'unanimité dans l'Assemblée (Très bien! Très bien! - Rires). Car, enfin, que poursuivons-nous? L'union de tous les Français autour d'un même drapeau, autour d'une même loi, autour d'un même pacte, et le jour où on consultera de nouveau le pays, dans quatre ans, quand il aura passé cet espace de temps dans la paix, dans le travail et dans l'ordre public, la majorité augmentera singulièrement; nous agirons d'ici là par nos actes et nos votes pour qu'elle atteigne l'unanimité [...]

Par conséquent, les moyens employés jusqu'à ce jour doivent être de nouveau mis en œuvre pour développer, augmenter, accroître cette majorité jusqu'à ce qu'elle embrasse le pays tout entier pour en faire un seul faisceau, pour être vraiment la France une et indivisible (Marques générales d'assentiment. - Bravos). (Gambetta, 1881, V, p. 191)

Les quantités indéfinies prennent ici des formes nominalisées : «la majorité », «la plus grande partie», "le chiffre élevé» et... "l'unanimité», seule en mesure d'assurer l'union, celle de «la France une et indivisible ». L'utilisation de ces quantités révèle une conception quelque peu particulière de la démocratie. Et pourtant, d'après le sténographe, ce public rouge lyonnais semble approuver. Léon Gambetta, le leader opportuniste, y est venu défendre une constitution très critiquée, au nom du principe des réformes progressives. Les risques de restauration monarchique sont encore présents et la révision constitutionnelle n'est pas d'actualité.

La première utilisation d'une quantité nominalisée se veut tout à la fois stigmatisante et unifiante : il convient de se moquer des ennemis de la République. Le sténographe note les rires de la salle. Pour autant, il n'est pas question d'une «République une et indivisible», mais d'une «France une et indivisible ». Le choix de l'énoncé, véritable sacralisation de l'Un, révèle la dimension performative du discours : quelles que soient les divisions sur la forme répu- 
blicaine, nul ne saurait contester l'unité nationale. La République n'est autre que la France, représentée par les trois couleurs du drapeau français (Girardet, 1984, p. 10), véritable bataille symbolique contre les «blancs» royalistes et les «rouges» internationalistes.

Dans l'ensemble de notre corpus, plusieurs syntagmes nominaux sont régulièrement utilisés : «un pays tout entier», «un peuple tout entier», «une nation tout entière », "une France tout entière ». Ce choix énonciatif réalise linguistiquement un acte de langage au service d'une stratégie politique inscrite dans l'histoire républicaine. Le « tout entier », qu'il soit peuple ou nation, s'oppose aux visées idéologiques issues des débats de la «grande Révolution », ou encore de ceux de 1848 puis, sous le Second Empire, celui d'une représentation ouvrière. Le Un, dans l'argumentation de ces fondateurs, ne peut accepter aucune forme de particularisme.

Le dictionnaire Littré s'inscrit dans cet événement discursif. À l'article «UnUne» (édition 1863-1877, Vl, p. 6542-6545), sur les vingt-deux points et seize dérivés, il n'est pas fait mention une seule fois de la République. Seule la France est Une. Littré, pourtant fier républicain, préfère laisser décliner l'histoire longue de France à propos du Un. En revanche, à l'article «Indivisible» (édition 1863-1877, III, p. 3189), il est fait référence à la République : « la République est une et indivisible, titre que prit la République française, par opposition aux tendances fédéralistes ». Le dictionnaire évoque un titre et non un principe. Il en fait même une forme de simple réaction à un contexte historique, celui de l'éclatement fédéraliste.

Au sein de cet événement discursif fondateur, se matérialisent à propos du nombre Un, les difficultés à penser tout à la fois la rupture avec l'Ancien Régime (société de corps), l'héritage révolutionnaire (accepté ou non comme un bloc) et la modernité démocratique (les millions d'atomes de souveraineté).

Pour conclure, nombres et chiffres sont très largement porteurs d'une valeur argumentative dans les discours fondateurs de la Troisième République. Vertus légitimante, stigmatisante, unifiante, clivante s'entremêlent dans la matérialité de la langue pour développer l'horizon d'attente d'une République Une et indivisible. Le marquage linguistique que constituent les nombres, pour ces orateurs républicains dont la plupart appartiennent à des loges maçonniques particulièrement sensibles à leur symbolique, contribue à la force illocutionnaire de leur discours. Analyser la performativité de ces discours amène l'historien à nouer ensemble l'étude du contexte langagier de fondation de la République et celle des actes de langage agissant sur ce contexte dans l'interaction entre leaders et publics populaires. Suivant les conclusions de Roger Chartier sur les origines culturelles de la Révolution française, il convient en effet d'insister sur le rôle des publics, qui ne sont en rien réceptacle ou cire molle (Chartier, 1990, p. 30). 
Tout récemment, qu'ont donc entendu les milliers de spectateurs et de téléspectateurs, le 14 juillet 2012, lorsque le discours de Gambetta du 14 juillet 1872 a résonné place de la Concorde, en ouverture du défilé militaire devant la tribune du tout nouveau président de la République François Hollande? Il conviendrait d'analyser cette situation de communication dans le cadre d'une sociologie des usages (Le Grignou, 2003). Pourquoi avoir choisi le discours de Gambetta du 14 juillet 1872 à la Ferté-sous-Jouarre et non celui du 14 juillet 1880 à Paris, première fête nationale officielle d'une République installée? Ces 140 ans d'écart entre 1872 et 2012 gomment de fait les longs débats houleux de 1880 sur le concept de «fête nationale » et les hésitations sur le choix symbolique d'une date commémorative (Amalvi, 1984, p.423). L'on retrouve donc, en 2012, cette dimension performative de l'unité incarnée dans une date bicéphale. Sont commémorés conjointement, depuis 1880, le 14 juillet 1789 et le 14 juillet 1790, une prise de la Bastille (mythiquement populaire et sanglante) et une fête de la fédération (œcuménique et officielle), soit un compromis se cachant derrière deux dates pour un même nombre : le $14 \mathrm{du}$

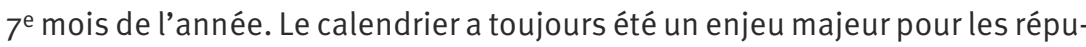
blicains malgré l'échec du calendrier dit « révolutionnaire».

En d'autres termes, chiffres et nombres font partie intégrante de l'histoire discursive républicaine française. Et si l'on veut bien s'y arrêter un instant, peu d'histoires nationales peuvent ainsi se décliner entre Première, Deuxième, Troisième, Quatrième, Cinquième République, sans compter les débats actuels sur la Sixième. Comme concluait ironiquement Prévert dans son poème Les belles familles:

\author{
Louis XVIII \\ et plus personne plus rien... \\ qu'est-ce que c'est que ces gens-là \\ qui ne sont pas foutus \\ de compter jusqu'à vingt?
}

\title{
Corpus
}

BLANC Louis, 1882, Discours politiques, 1847-1881, Paris, Germer-Baillière.

GAMBETTA Léon, 1881, Discours et plaidoyers politiques de Gambetta, rassemblés par J. Reinach, Paris, Charpentier (11 volumes).

Floquet Charles, 1885, Discours et opinions, rassemblés par A. Faivre, Paris, Derveaux

(2 volumes).

Hugo Victor, 1968, Actes et Paroles, Euvres complètes de Victor Hugo présentées par

J.-L. Cornuz, Paris, Rencontre.

LITTRÉ Émile, 1863-1877, Dictionnaire de la langue française, Paris, Hachette (5 volumes). 


\section{Références}

Amalvı Christian, 1984, "Le 14 juillet. Du Dies irae à Jour de fête », Les lieux de mémoire, P. Nora éd., Paris, Gallimard, p.383-422.

Amossy Ruth, 2000, L'argumentation dans le discours. Discours politique, littérature d'idées, fiction, Paris, Nathan.

Aprile Sylvie, 2005, "Louis Blanc, un des pères fondateurs de la "vraie République" », Louis Blanc, un socialiste en République, F. Demier éd., Paris, Creaphis, p. 171-182.

Branca-Rosoff Sonia, Guilhaumou Jacques, 2003, «De société à socialisme. L'invention néologique et son contexte discursif», Dictionnaire des usages socio-politiques, 1770-1815, Paris, Champion, fascicule VII, p. 39-77.

Charaudeau Patrick, 2000, «Une problématisation discursive de l'émotion. À propos des effets de pathémisation à la télévision », C. Plantin et al., Les émotions dans les interactions, Lyon, Arci / Presses universitaires, p. 125-155.

ChARTIER Roger, 1990, Les origines culturelles de la Révolution française, Paris, Seuil.

DAYAN Daniel, 1992, "Les mystères de la réception», Le Débat, n71, septembreoctobre, p. 146-162.

DUCROT Oswald, 1984, Le dire et le dit, Paris, Minuit.

FEYEL Gilles, 2007, La presse en France des origines à 1944. Histoire politique et matérielle, Paris, Ellipses (Infocom).

GARDEY Delphine, 2008, Écrire, calculer, classer. Comment une révolution de papier a transformé les sociétés contemporaines (1800-1940), Paris, La Découverte.

GIRARDEt Raoul, 1984, "Les trois couleurs. Ni blanc, ni rouge», Les lieux de mémoire, P. Nora éd., Paris, Gallimard, p. 49-66.

Guilhaumou Jacques, 2006, Discours et événement. L'histoire langagière des concepts, Besançon, Presses universitaires de Franche-Comté.

KaLIFA Dominique, 2001, La culture de masse en France, Repères, La Découverte.

Kerbrat-Orecchion Catherine, 2002, "Rhétorique et interaction», Après Perelman : quelles politiques pour les nouvelles rhétoriques? L'argumentation dans les sciences du langage, R. Koren, R. Amossy éd., Paris, L'Harmattan, p. 173-196.

KoSELLECK Reinhart, 1990, Le futur passé. Contribution à la sémantique des temps historiques, Paris, EHESS.

KRIEg-PlANQUe Alice, 2011, «Les “petites phrases”. Un objet pour l'analyse des discours politiques et médiatiques», Les "petites phrases» en politique, C. OllivierYaniv, A. Krieg-Planque éd., Communication et Langages, nº 168, p. 23-41.

LE GRIGnOu Brigitte, 2003, Du côté du public. Usages et réceptions de la télévision, Paris, Economica.

LEQUIN Yves, 1977, Les ouvriers de la région lyonnaise, 1848-1914. La formation de la classe ouvrière régionale, Lyon, Presses universitaires de Lyon.

MAILLET Jean, 2011, «Attendre 107 ans »... et toutes les autres expressions qui comptent, Paris, L'Opportun.

Maingueneau Dominique, Cossuta Frédéric, 1995, "L'analyse des discours constituants », Langages, nº 117, p. 112-125. 
Nicolet Claude, 1982, L'idée républicaine en France. Essai d'histoire critique, Paris, Gallimard.

Ollivier-YAniv Caroline, KRIEg-PLAnque Alice éd., 2011, Les «petites phrases» en politique, Communication et Langages, n ${ }^{0} 168$.

Perrot Michelle, 1984, Jeunesse de la grève, Paris, Seuil.

Rosanvallon Pierre, 1998, Le peuple introuvable. Histoire de la représentation démocratique en France, Paris, Gallimard.

SEARLE John R., 1982, Sens et expression, trad. et préf. J. Proust, Paris, Minuit. 